\title{
Searches for vector-like quarks and ttbar resonances with the ATLAS detector
}

\author{
Jordan S WEBSTER* \\ On behalf of the ATLAS Collaboration \\ University of Chicago (US) \\ E-mail: jordan.s.webster@cern.ch
}

\begin{abstract}
Several extensions of the Standard Model predict the existence of exotic resonances that couple strongly to the top quark. These proceedings report on ATLAS searches for high mass top-antitop resonances in the lepton plus jets and fully hadronic decay channels. Also presented are ATLAS searches for pair-produced vector-like quarks decaying to a Higgs boson plus a top quark or a $W$ boson plus a $b$ quark, and singly produced vector-like quarks decaying to a $W$ or $Z$ boson plus a light quark. The searches use subsets of the $p p$ collision data recorded at the LHC in 2011 and 2012 at centre-of-mass energies equal to $7 \mathrm{TeV}$ and $8 \mathrm{TeV}$, respectively. Results are found to be consistent with the Standard Model, and limits are set on the production of narrow $t \bar{t}$ resonances and vector-like quarks.
\end{abstract}

XXI International Workshop on Deep-Inelastic Scattering and Related Subject -DIS2013, 22-26 April 2013

Marseilles, France

\footnotetext{
* Speaker.
} 


\section{Introduction}

Due to its unique properties, the top quark is a central focus in many beyond the Standard Model (BSM) theories. Since it is by far the most massive of the known quarks, new strong dynamics often couples most strongly to the top. It also has a distinctive experimental signature, since unlike the lighter quarks it decays to a $W$ boson and a $b$ quark before it has time to hadronize. Thus, searching for resonances in events with top quarks is one of the many undertakings of the ATLAS Collaboration.

This note focuses on ATLAS searches for BSM production of $t \bar{t}, H t+W b$, and $W / Z+$ jets. Constraints on $t \bar{t}$ production are interpreted in the context of benchmark models that predict a narrow resonance $[1,2]$. Constraints on the latter production processes are interpreted in the context vector-like quarks (VLQs) [3, 4], which are predicted in extra-dimensional models, grand unification theories based on E6, and the little higgs models.

\section{2. $t \bar{t}$ resonance searches}

ATLAS $t \bar{t}$ resonance searches are conducted in the lepton plus jets $(\ell+$ jets $)$ and fully hadronic decay channels, using two models as benchmarks. The first model is topcolor assisted technicolor [1], which predicts a leptophobic $Z^{\prime}$ that decays to $t \bar{t}$ about a third of the time for masses above $700 \mathrm{GeV}$. The second is a Randall-Sundrum warped extra dimension model [2], which predicts a Kaluza-Klein gluon (KK-g) that has a branching fraction to $t \bar{t}$ of roughly $90 \%$.

\subsection{Lepton plus jets $t \bar{t}$ search}

The search for a $t \bar{t}$ resonance decaying to $\ell+$ jets uses $4.7 \mathrm{fb}^{-1}$ of $7 \mathrm{TeV}$ ATLAS data [5]. The analysis is divided into resolved and boosted categories. The former consists of events in which the hadronic top decay produces three separate jets, reconstructed with a radius parameter $R=0.4^{1}$. When the hadronic top is sufficiently boosted, however, the three jets from the decay can merge into a single fat jet with a radius $R \approx 1.0$. Such events are put into the boosted category.

Selected events are required to have exactly one isolated lepton and missing transverse momentum $\left(E_{\mathrm{T}}^{\text {miss }}\right)$ greater than 20 to $35 \mathrm{GeV}$ depending on the lepton flavor. Events passing the boosted selection must have at least one fat jet and a resolved jet within $\Delta R=\sqrt{\Delta \phi^{2}+\Delta \eta^{2}}=1.5$ of the lepton. The resolved selection requires at least three resolved jets (four if none of the jets have a mass above $60 \mathrm{GeV}$ ). Both selections require there to be at least one $b$-tagged jet in the event. The dominant background comes from SM $t \bar{t}$ production, which is modeled using Monte Carlo (MC) simulated data samples. Reducible backgrounds from single top, $W / Z+$ jets, and diboson production are modeled primarily by $\mathrm{MC}$, with the exception of the $W+$ jets normalization, which is calculated using the charge asymmetry in $W$ production measured in data. The multijet background is estimated from data using the matrix method.

The invariant mass of the $t \bar{t}$ system is used to search for signal. To calculate this mass, jets need to be assigned to the $t \bar{t}$ system. The best assignment is chosen by minimizing a $\chi^{2}$ algorithm, which is found to achieve the correct parton assignment for roughly $65 \%$ of events in which all

\footnotetext{
${ }^{1}$ Details regarding the ATLAS detector and coordinate system can be found in Ref. [6].
} 

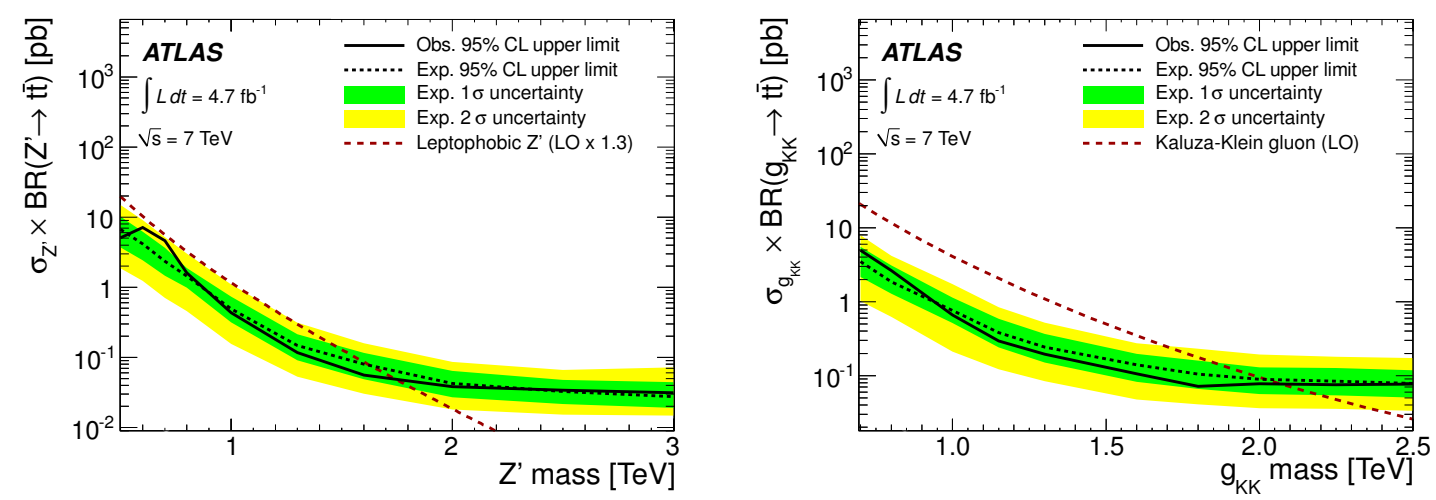

Figure 1: Observed and expected 95\% confidence level upper cross section limits for the $Z^{\prime}$ (left) and KK-g (right) resonances from the semi-leptonic $t \bar{t}$ analysis [5].

decay products can reconstructed. No signal is observed in either of the selection categories, so upper limits are set on the cross section of a narrow $t \bar{t}$ resonance, as shown in Fig. 1. For the $Z^{\prime}$, masses are excluded between 0.5 and $1.7 \mathrm{TeV}$, and for the KK-g, masses are excluded between 0.5 and $1.9 \mathrm{TeV}$.

\subsection{Fully hadronic $t \bar{t}$ search}

The corresponding fully hadronic search using the same ATLAS dataset looks for events where both top quarks decay to fat jets [7]. Two different taggers are employed to tag jets from hadronic top decays. The HEPTopTagger [8] reclusters fat jets into triplets of subjets, and then checks if each triplet is compatible with a hadronic top decay. Alternatively, the Top Template Tagger [9] compares the fat jet topology to a library of roughly $300 \mathrm{~K}$ templates and checks for matches. Events passing the HEPTopTagger (Top Template Tagger) selection must have at least two top-tagged fat jets with a radius $R=1.5(1.0)$ and $p_{\mathrm{T}}>200 \mathrm{GeV}(450 \mathrm{GeV})$, each with an associated $b$-tagged jet within a cone of radius $\Delta R=1.4(1.0)$. In this search, the systematic uncertainty due to the $b$-tagging efficiency can be as large as $50 \%$ (for events with jets that have $p_{\mathrm{T}}>800 \mathrm{GeV}$ ) due to the limited understanding of dense tracking environments.

The dominant backgrounds are associated with $t \bar{t}$ and multijet events. The $t \bar{t}$ component is generated by MC and the multijet component is calculated from data via a control region extrapolation. No signal is observed in the $t \bar{t}$ mass distribution, so upper limits are set on the cross section of a narrow resonance. Combining results from both taggers, masses are excluded for the $Z^{\prime}$ from 0.7 to $1.00 \mathrm{TeV}$ and 1.28 to $1.32 \mathrm{TeV}$. For the KK-g, masses are excluded from 0.7 to $1.62 \mathrm{TeV}$.

\section{Vector-like quark searches}

Vector-like quarks are defined as quarks with left- and right-handed components that undergo the same transformations under SU(2) [4]. Different production mechanisms are favored by different classes of VLQ models. The text below summarizes separate searches for strongly produced VLQ pairs and weakly produced VLQs. 


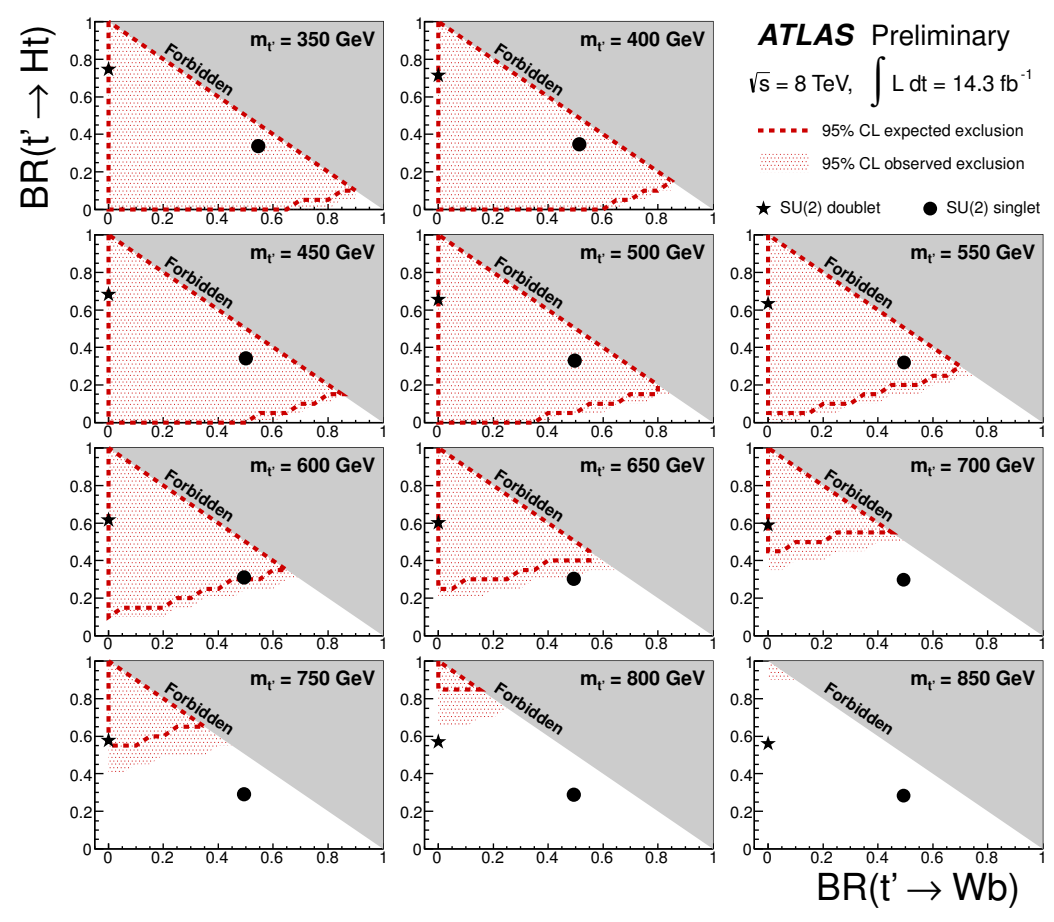

Figure 2: Observed and excluded 95\% confidence level exclusions from the pair-produced VLQ search [10], shown with respect to $B R\left(t^{\prime} \rightarrow W b\right)$ vs. $B R\left(t^{\prime} \rightarrow H t\right)$. The black circle and star represent two benchmark VLQ models.

\subsection{Pair produced VLQs}

Due to precision electroweak measurements, VLQs are typically only allowed to mix with the third generation [3]. In this construction, strong pair production dominates for VLQs with masses less than a TeV. Such VLQs decay to $H t, Z t$, or $W b$. The ATLAS search for pair-produced VLQs uses $14.3 \mathrm{fb}^{-1}$ of $8 \mathrm{TeV}$ data [10]. The analysis focuses on decays to $H t+W b$, where the $H$ goes to $b \bar{b}$ and the $W$ decays leptonically. Selected events are required to have exactly one isolated lepton, $E_{\mathrm{T}}^{\mathrm{miss}}>20 \mathrm{GeV}$, and at least 6 jets where at least two of the jets are $b$-tagged.

Many background processes are considered in the description of this final state, including $t \bar{t}+$ jets, $W / Z+$ jets, single top, $t \bar{t} V, t \bar{t} H$, diboson, and multijet production. All of these components come from MC, with the exception of the multijet component and the $W+$ jets normalization, which are both calculated from data using the same methods applied in the $t \bar{t}$ resonance search from Section 2.1. Signal events for a $t^{\prime}$ resonance are generated using MC. The distribution of the scalar sum of all transverse momentum in the event, $H_{\mathrm{T}}$, is used to search for signal, since calculating the invariant mass of the VLQ is difficult with this final state. Since no signal is observed, upper limits are put on the $t^{\prime}$ production cross section as a function of the mass and the $H t$ and $W b$ branching ratios, as shown in Fig. 2. For a weak-isospin singlet model, $t^{\prime}$ masses are excluded from 350 to $640 \mathrm{GeV}$. For a weak-isospin doublet model, which has a much smaller branching ratio to $\mathrm{Wb}$, masses are excluded from 350 to $790 \mathrm{GeV}$. 


\subsection{Singly produced VLQs}

Despite precision EWK measurements, it is possible for VLQs to couple to light quarks if symmetries cause the light quark couplings to vanish [4]. In this construction, weak single production becomes dominant and the VLQ typically decays to a $W$ or $Z$ boson plus a light quark. A small enhancement of single top production is predicted, but top quarks are not the primary focus.

The ATLAS search for such VLQs uses $4.64 \mathrm{fb}^{-1}$ of $7 \mathrm{TeV}$ data [11]. The selection requires a leptonically decaying $W$ or $Z$ boson plus two or more jets, where at least one of the jets has $p_{\mathrm{T}}>60 \mathrm{GeV}$. Along with the reconstructed vector boson, the jet with the highest $p_{\mathrm{T}}$ is used to define the VLQ system. A background model is generated by fitting a smooth function to the invariant mass of this VLQ system in data. No signal is observed, so upper cross section limits are set on the associated production of a resonance decaying to a vector boson and a jet. Signal templates are generated using MC for the VLQ resonances predicted in Ref. [4], including a downtype $D$ quark and up-type $U$ quark. For a particular choice of coupling $(\tilde{\kappa}=1), D$ quarks are excluded with masses from 400 to $1120 \mathrm{GeV}$ and $U$ quarks are excluded with masses from 400 to $1080 \mathrm{GeV}$.

\section{Conclusion}

Four ATLAS analyses are presented, which constrain benchmark models for enhanced top production at the LHC. The $t \bar{t}$ invariant mass spectrum is probed to over $3 \mathrm{TeV}$ and limits are set on the production cross section for a narrow resonance. Searches for VLQs put limits on BSM production of $H t+W b$ and $W / Z+$ jets. These searches employ advanced analysis techniques that rely on multivariate methods like hadronic top-tagging and $b$-tagging. Such tools are found to be robust, expanding the possibility for creativity in future searches for top quark decays.

\section{References}

[1] C. T. Hill, Phys.Lett. B345 (1995) 483-489, arXiv:hep-ph/9411426 [hep-ph].

[2] B. Lillie, L. Randall, and L.-T. Wang, JHEP 0709 (2007) 074, arXiv:hep-ph/0701166 [hep-ph].

[3] J. Aguilar-Saavedra, JHEP 0911 (2009) 030, arXiv: 0907.3155 [hep-ph].

[4] A. Atre, G. Azuelos, M. Carena, T. Han, E. Ozcan, et al., JHEP 1108 (2011) 080, arXiv: 1102 . 1987 [hep-ph].

[5] ATLAS Collaboration, arXiv: 1305.2756 [hep-ex].

[6] ATLAS Collaboration, JINST 3 (2008) S08003.

[7] ATLAS Collaboration, JHEP 1301 (2013) 116, arXiv: 1211.2202 [hep-ex].

[8] ATLAS Collaboration, Tech. Rep. ATLAS-CONF-2012-065, Jun, 2012.

[9] L. G. Almeida, S. J. Lee, G. Perez, G. Sterman, and I. Sung, Phys.Rev. D82 (2010) 054034, arXiv: 1006.2035 [hep-ph].

[10] ATLAS Collaboration, Tech. Rep. ATLAS-CONF-2013-018, CERN, Geneva, Mar, 2013.

[11] ATLAS Collaboration, Tech. Rep. ATLAS-CONF-2012-137, CERN, Geneva, Sep, 2012. 